\title{
A Model of Electro-Kinetic Platform based on Printed Circuit Board Technology for Manipulation and Detection of Liver Cancer Cells
}

\author{
Reda Abdelbaset \\ Biomedical Engineering \\ Department, Helwan University, \\ Cairo, Egypt. \\ Center of Nano electronics and \\ Devices (CND), Zewail City of \\ Science and Technology and \\ The American University in \\ Cairo (AUC), Egypt.
}

\author{
Yehya H. Ghallab \\ Biomedical Engineering \\ Department, Helwan University, \\ Cairo, Egypt. \\ Center of Nano electronics and \\ Devices (CND), Zewail City of \\ Science and Technology and \\ The American University in \\ Cairo (AUC), Egypt.
}

\author{
Hamdy Abdelhamid, \\ Yehea Ismail \\ Center of Nano electronics and \\ Devices (CND), Zewail City of \\ Science and Technology and \\ The American University in \\ Cairo (AUC), Egypt.
}

\begin{abstract}
This paper presents a model of an electrokinetic platform which combines three configurations (traveling, electrorotation, and levitation) for manipulation, characterization and separation of liver cancer cells based on dielectrophoresis phenomena. The dielectrophoresis phenomena is a motion of uncharged polarizable particles towards the location of extreme field strength in a non-uniform electric field. Recently, a dielectrophoresis (DEP) became a prominent technique for manipulation and characterization of biological particles. The traveling-wave dielectrophoresis (twDEP), Electro-rotation and levitation are electro-kinetic methods which are produced by the interaction between a non-uniform electric field and polarizable particles. In this work, a 2D model of three configurations of electro-kinetic platform based on Printed Circuit Board technology (PCB) for differentiating between liver cancer cells and normal liver cells is presented and discussed.
\end{abstract}

\section{Keywords}

Dielectrophoresis, Traveling, Electro-rotation, Levitation, Liver cancer cells and COMSOL

\section{INTRODUCTION}

The dielectrophoresis (DEP) is widely used for manipulation and characterization of biological cells by monitoring and characterizing the response of biological cells towards a nonuniform electric field, where a non-uniform electric field generates a net force on the field-induced dipole of a particle [1-4].

There are several advantages make DEP phenomena is most commonly used in the field of manipulation and characterization such as 1) its ability to manipulate and characterize the biological with high efficiency, where the dielectric properties of biological particles within a specific range of frequencies of an electric field can characterize cells. 2) cheaper because it does not need any expensive reagents like other techniques.

The DEP force is the induced force on an uncharged particles as a resultant of a non-uniform electric field which is generated by specific design of microelectrodes. Furthermore, this force depends on the properties of applied electric field i.e. frequency, amplitude and waveform), the size of particles and the dielectric properties of the particles compared to the surrounding medium [5-6]. The PCB technology is chosen due to several advantages such as low cost, widely available, re-workable and excellent shelf life [7]. Liver cancer is the sixth most common cancer in the world, with 782,000 new cases diagnosed in 2012 [8]. There are many reasons of liver cancer such as Hepatitis B and hepatitis C viruses [8], therefore, it is important to provide a new technique to detect this cancer.

The main objective of this paper is to prove the capability of the proposed electrokinetic platform based on PCB technology to distinguish the liver cancer cells from normal liver cells. The rest of paper is arranged as follows: Section 2 explains the theory of dielectrophoresis phenomena. Section 3 presents the structure of the proposed electrokinetic platform (twDEP, electro-rotation and levitation microelectrodes). Section 4 presents a simulation model of the proposed platform and biological particles (liver cancer and normal cells). The simulation results of DEP forces, the acquired kinetic energy and the velocity of each type of particles (liver cancer and normal cells) are stated in Section 5. Finally, section 6 concludes this paper and summarizes the advantages of the presented model based on the simulation results.

\section{THEORY OF OPERATION}

The DEP phenomena describes the generated force as a result of the interaction between a non-uniform electric field and polarizable particles. The governing vector relationship defining the DEP force is: FDEP $=(\rho \cdot \nabla) \mathrm{E}$, where $\rho$ is the effective polarization induced in the particle, $\nabla$ is the gradient operator and $\mathrm{E}$ is non-uniform electric field [9].

The non-uniform electric field is generated, when a sequence of signals shifted by $90^{\circ}$ phase is applied to a microelectrodes array. Near the electrode surface, a particle may undergo a translation movement. A particle subjected to an electric field of a traveling wave will have an induced force by the field [10]:

$\mathrm{FDEP}=2 \pi \varepsilon_{o} \varepsilon_{m} \mathrm{R}^{3}\left[\operatorname{Re}(\mathrm{Ke}) \nabla \mathrm{E}^{2}+2 \operatorname{Im}(\mathrm{Ke}) \nabla \times\left(E_{I} \times E_{R}\right)\right]$ (1),

$\mathrm{E}=E_{R}+\mathrm{j} E_{I}(2)$,

where, $\varepsilon_{0}$ is the permittivity of free space, $\varepsilon_{\mathrm{m}}$ is the relative permittivity of the surrounding medium, $R$ is the radius of particle, $E_{R}$ is the real part of electric field, $E_{I}$ is the imaginary 
part of electric field, Im is the imaginary part, Re is the real part of $\mathrm{CM}$ and $[\mathrm{Ke}]$ is the Clausius-Mossotti factor $(\mathrm{CM}$ factor). The CM factor can be calculated such as [10]:

$\operatorname{Ke}(\boldsymbol{\omega})=\frac{\varepsilon_{\boldsymbol{p}-}^{*} \varepsilon_{\boldsymbol{m}}^{*}}{\varepsilon_{\boldsymbol{p}+2}^{*} \varepsilon_{\boldsymbol{m}}^{*}}(3)$,

$\varepsilon_{p}^{*}=\varepsilon_{p}-\frac{i \sigma_{p}}{\omega}, \varepsilon_{m}^{*}=\varepsilon_{m}-\frac{i \sigma_{m}}{\omega}$

where $\varepsilon_{\mathrm{p}}, \varepsilon_{\mathrm{m}}$ are the permittivity of particle and medium and $\sigma_{\mathrm{p}}$ and $\sigma_{\mathrm{m}}$ the conductivity of particle and medium, respectively, $\omega$ is the angular frequency of the electric field, Therefore, the CM factor depends on the permittivity and conductivity of the medium and the characteristics of the particle (cell membrane and cytoplasm), in addition to the frequency of the applied electric field. These variables have capability to change the sign and the magnitude of CM factor, which would change the direction and scale down the magnitude of DEP force.

The main idea of levitation is creating area at which the electric field being weaker than the electric field at surrounding areas. This principle is achieved when specific signal applied on electrodes which would be explained in the next sections. A particle subjected to an induced force by the field [10]:

$\mathrm{FDEP}=2 \pi \varepsilon_{m} \mathrm{R}^{3} \operatorname{Re}[\mathrm{Ke}] \nabla \mathrm{E}^{2}$

The electro-rotation is achieved when a rotating electric field is applied on polarizable particle, where a dipole moment is induced on particle as result of electric field [10],

$P=4 \pi \varepsilon_{m} \mathrm{R}^{3} \mathrm{KeE}(6)$,

Afterwards, the dipole moment starts to rotate in conjunction with the electric field but with some lag by a phase factor related to the complex. A torque would be generated on particles as a result of rotating electric field and induced dipole moment [10]

$\mathrm{T}=-4 \pi \varepsilon_{m} \mathrm{R}^{3} \operatorname{Im}[\mathrm{Ke}] \mathrm{E}^{2}(7)$.

A model of a liver cell in a saline medium is shown in Figure 1. the difference between the liver cancer and normal liver cells in this model is the dielectric properties of a cell. However, the medium is constant but the change in dielectric properties of particles will cause a change in the value of Clausius-Mossotti factor (Ke), consequently, causes a change in the induced DEP force on particles.

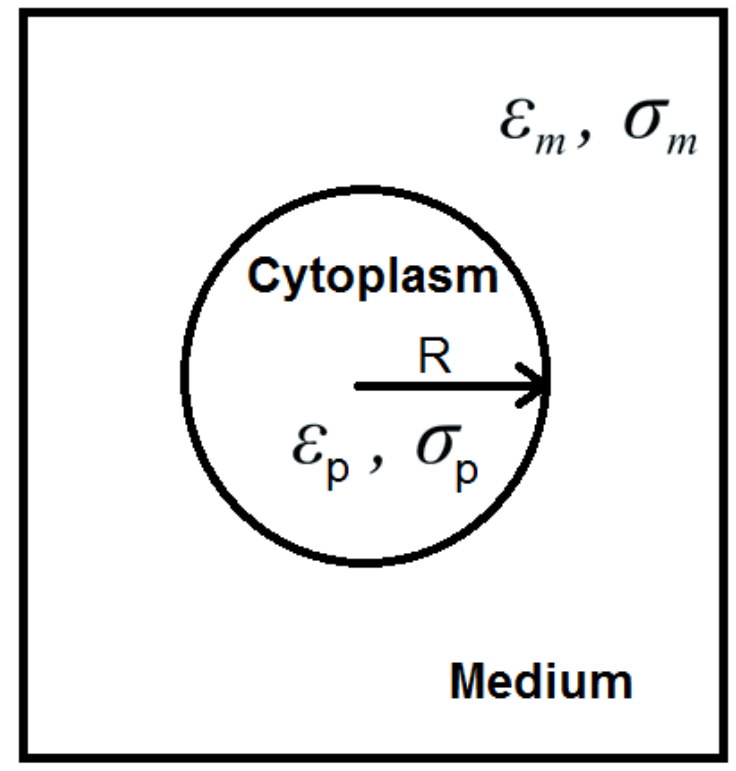

Figure 1: A model of liver cells: the medium is saline $\left(\sigma_{m}=0.33 \frac{s}{m} \& \varepsilon_{m}=78.69[11]\right)$, for liver cancer cells $\left(\mathbf{R}=50 \mu \mathrm{m}[12], \sigma_{m}=0.67 \frac{\mathrm{s}}{\mathrm{m}} \& \varepsilon_{m}=58.5\right.$ [13] $)$ and for normal liver cells $\left(R=20 \mu \mathrm{m}[14], \sigma_{m}=0.57 \frac{s}{m} \& \varepsilon_{m}=\right.$ $53[13])$.

\section{THE PROPOSED ELECTRO- KINETIC PLATFORM}

The proposed electro-kinetic platform combines three configurations which are traveling, electro-rotation and levitation to be able to distinguish between biological cells through multimethod for more efficiency. The twDEP configuration microelectrodes array can be implemented using several shapes, 1) a concentric rings structure [15], 2) a planar linear interdigitated array [16]. The micro- electrodes for DEP levitation are categorized as three types: a cone plate levitation system, Ring dipole levitation system and a quadrupole levitation system [9]. The microelectrodes for electro-rotation mainly based on four poles to generate a rotating electric field, different shapes are used, i.e. concentric rings configuration [15], octode [17], arrows (quadrupole and octupole) [18]. The concentric rings design is preferred for microelectrodes of traveling and electro-rotation configurations due to several advantages such as permits designing long electrodes in a small area that leads to minimizing the size of the lab-on-a- chip platform. Furthermore, it increases the intensity of electric field due to its roundness. Furthermore, it is compatible with PCB technology. The quadruple design is preferred because of it is compatible with PCB technology, unlike other designs which are very complex.

\section{2D MODEL OF ELECTRO-KINETIC PLATFORM}

COMSOL Multiphysics 5.0 is preferred for simulating the twDEP microelectrodes and liver cancer and normal cells. The stages of the model will be illustrated in the following subsections, which contain a preprocessing stage including space dimension, applied physics and study, and a processing stage including the geometry, materials and applied physics conditions as shown in Figure 2 [19]. 


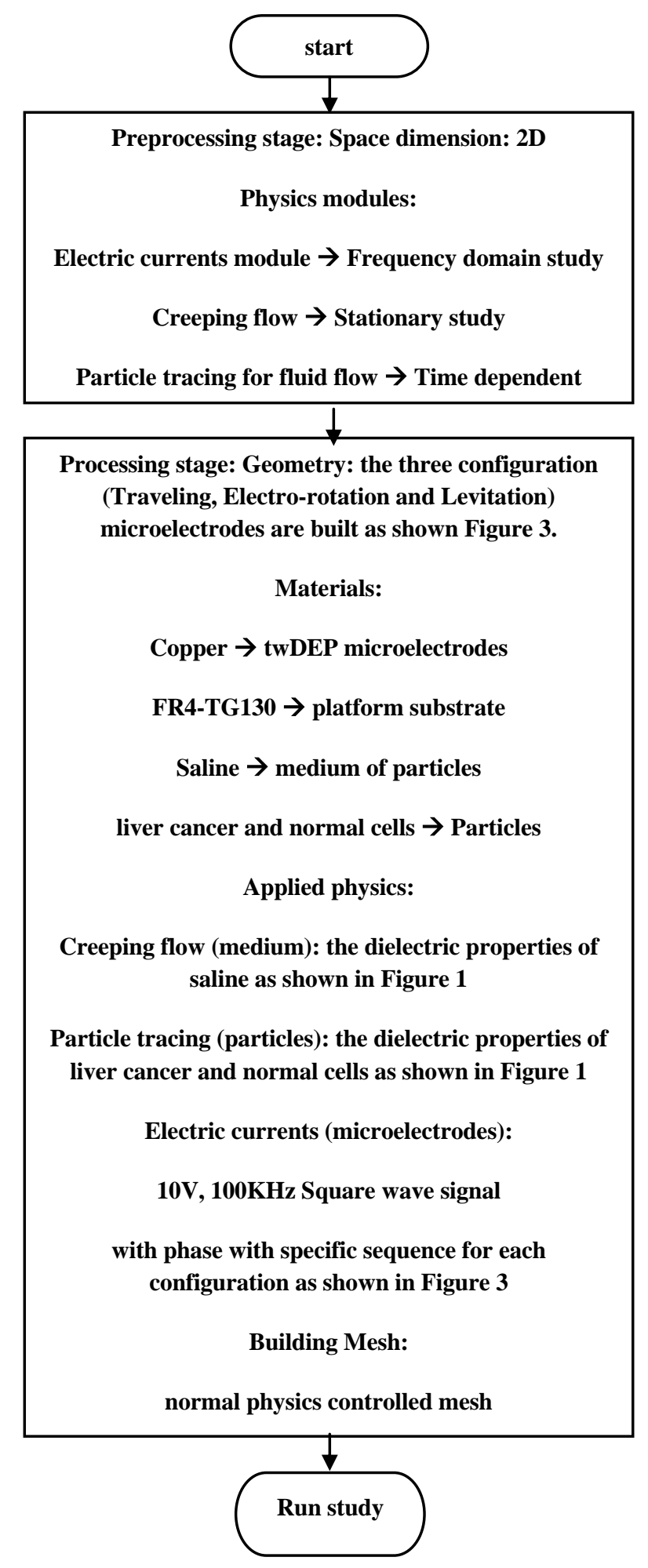

Figure 2: Flow Chart of COMSOL model.

The geometry of the three configurations of the proposed electro-kinetic platform is presented in Figure 3. The microelectrodes of traveling configuration are consisting of a four concentric ring (for simplifying the model but in real this four rings must have repeated more than one to get an efficient electric field) each ring has $150 \mu \mathrm{m}$ in width and $150 \mu \mathrm{m}$ in between two successive rings as shown in Figure 3 (A).

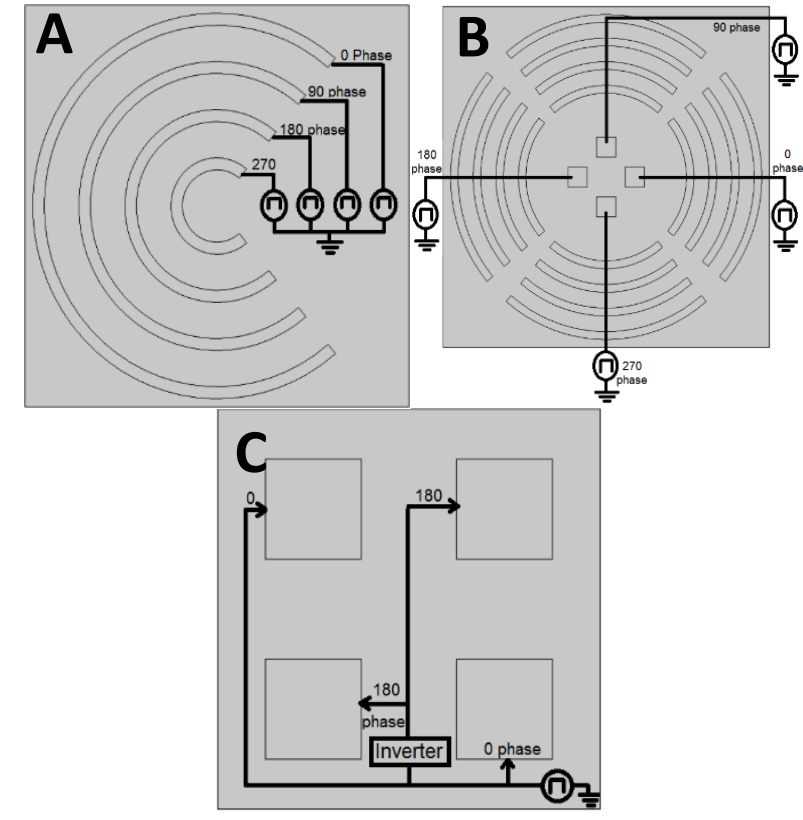

Figure 3: A geometry of the three configurations and the applied electrical potential, A) microelectrodes of traveling configuration, and B) microelectrodes of Electro-rotation configuration, and $\mathrm{C}$ ) microelectrodes of levitation configuration.

The microelectrodes of electro-rotation configuration are consisting of a four concentric ring (for simplifying the model but in real this four rings must have repeated more than one to get an efficient electric field) each ring has $150 \mu \mathrm{m}$ in width and divided into a four arcs and $150 \mu \mathrm{m}$ in between two successive rings as shown in Figure 3 (B). Furthermore, the concentric rings for electro-rotation configuration are supported by four poles to increase the intensity of electric field at center as shown in Figure 3 (B). The microelectrodes of levitation configuration are consisting of four poles each pole has $100 \mu \mathrm{m} \times 100 \mu \mathrm{m}$ size and $100 \mu \mathrm{m}$ in between every two poles as shown in Figure 3 (C).

\section{SIMULATION RESULTS}

In this part, the detailed results were presented to prove the ability of the proposed electro-kinetic platform based on PCB technology to detect and characterize the liver cancer cells from the normal liver cells. All figures are produced by COMSOL unless otherwise stated. The applied electrical potential on the three configurations of the electro-kinetic platform is presented in Figure 4, where the red of the color legend indicates to $100 \mathrm{KHz}$ square wave $10 \mathrm{v} 0^{\circ}$ phase, while the blue indicates to $100 \mathrm{KHz}$ square wave $10 \mathrm{v} 180^{\circ}$ phase, however, the green indicates to $100 \mathrm{KHz}$ square wave $10 \mathrm{v} 90^{\circ}$ phase and $270^{\circ}$ phase (is represented in the imaginary part, not zero). In addition to the electric field which is generated by the three configurations (traveling, electro-rotation and levitation) of the electro-kinetic platform is presented in Figure 4. The generated electric field is considered a good indicator of the efficiency of the electro-kinetic platform. 

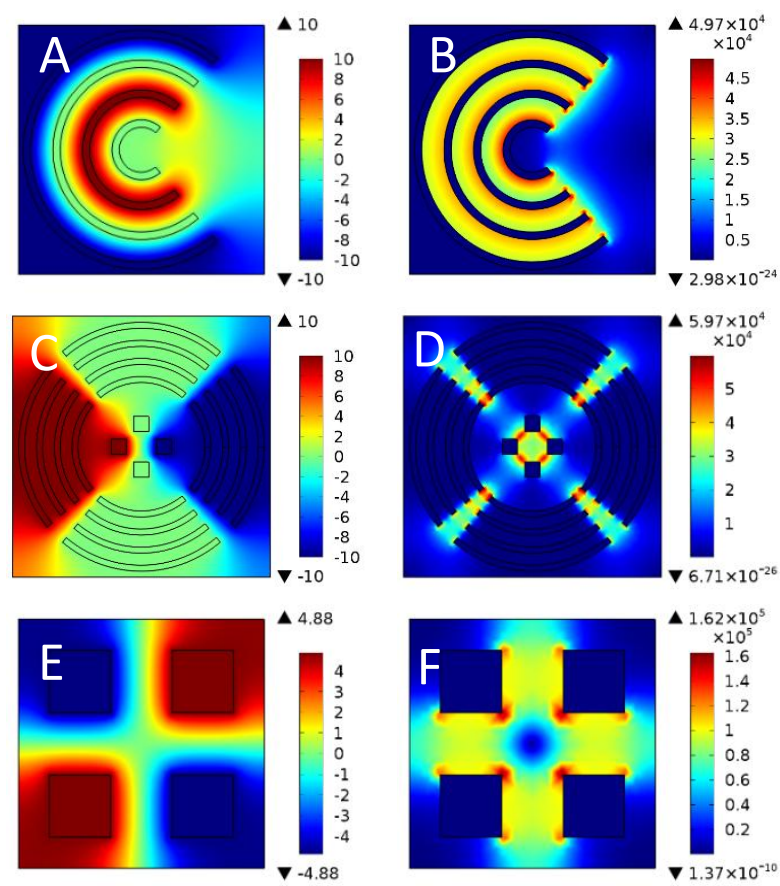

Figure 4: The applied electrical potential on: A) microelectrodes of traveling configuration, C) microelectrodes of electro-rotation configuration, and $\mathrm{E}$ ) microelectrodes of levitation configuration. The generated electric field of: B) microelectrodes of traveling configuration, D) microelectrodes of electro-rotation configuration, and F) microelectrodes of levitation configuration.

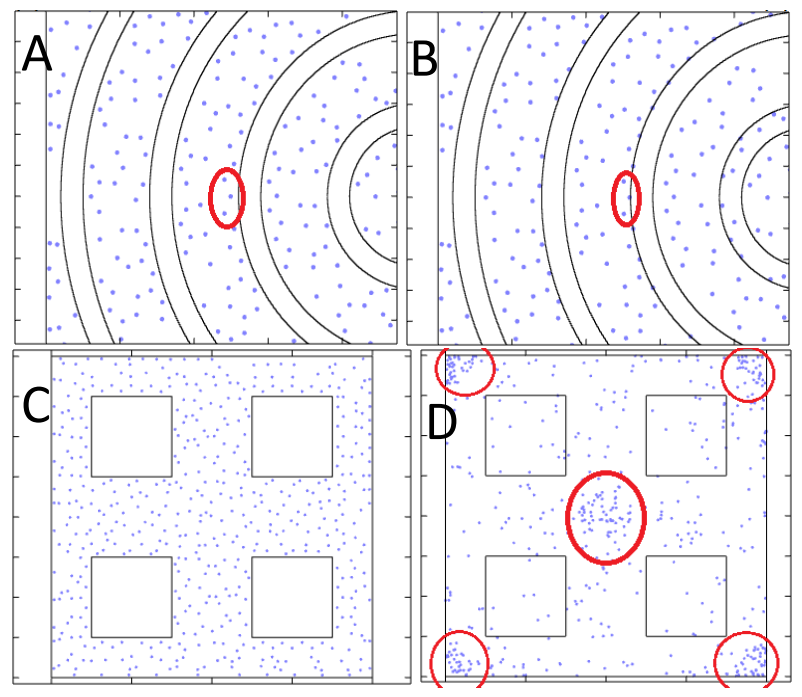

Figure 5: the spread of liver cells above microelectrodes of levitation configuration, A) random before applying electrical potential and B) trapped after applying electrical potential. The migration of liver cells above

microelectrodes of traveling configuration, $C$ ) before applying electrical potential and D) after applying electrical potential.

Both types of liver cancer cells acquired kinetic energy as a resultant of the induced DEP force which forced the cells to move over platform as shown in Figure 5. Figure 5 (A) and (B) shows the migration of liver cells above the microelectrodes of traveling configuration. While Figure 5 (C) and (D) shows the trapping of liver cells above the microelectrodes of levitation configuration. The rotation motion does not appear in the model due to the circular shape of liver cells. Figure 5 shows the ability of the proposed electro-kinetic platform to manipulate the liver cancer cells.

Each of Figure 6, Figure 7 and Figure 8 shows a comparison between the liver cancer cells and the normal liver cells in DEP force, kinetic energy and velocity which are generated by the three configurations of the proposed electro-kinetic platform to prove the ability of the proposed electro-kinetic platform to characterize and separate the liver cancer cells and normal liver cells.

- Figure 6 shows a comparison between the liver cancer cells and the normal liver cells in the induced twDEP force, the acquired kinetic energy and the velocity of migration of liver cells.

- Figure 7 shows a comparison between the liver cancer cells and the normal liver cells in the induced DEP rotation force, the acquired kinetic energy.

- Figure 8 shows a comparison between the liver cancer cells and the normal liver cells in the induced DEP levitation force, the acquired kinetic energy and the velocity of trapping of liver cells
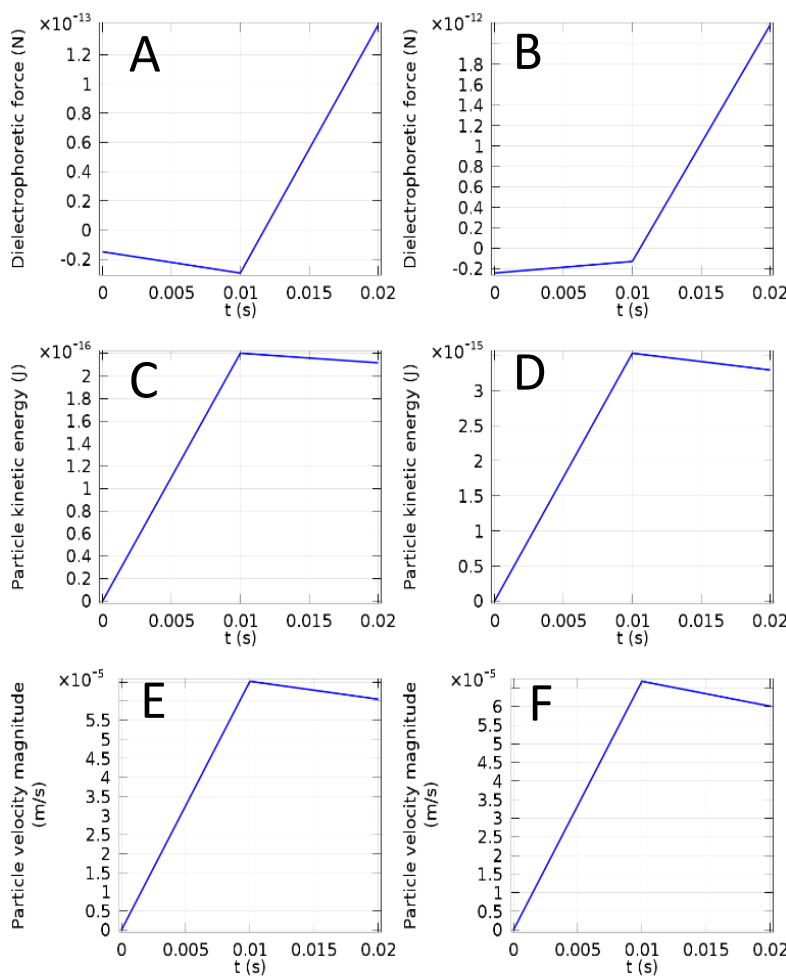

Figure 6: A comparison between the liver cancer cells and normal liver cells under effect of twDEP force in: A) the average induced DEP force on normal liver cells, B) the average induced DEP force on liver cancer cells, C) the average acquired kinetic energy by normal liver cells, D) the average acquired kinetic energy by liver cancer cells, E) the variance velocity of normal liver cells, $F$ ) the variance velocity of liver cancer cells. 

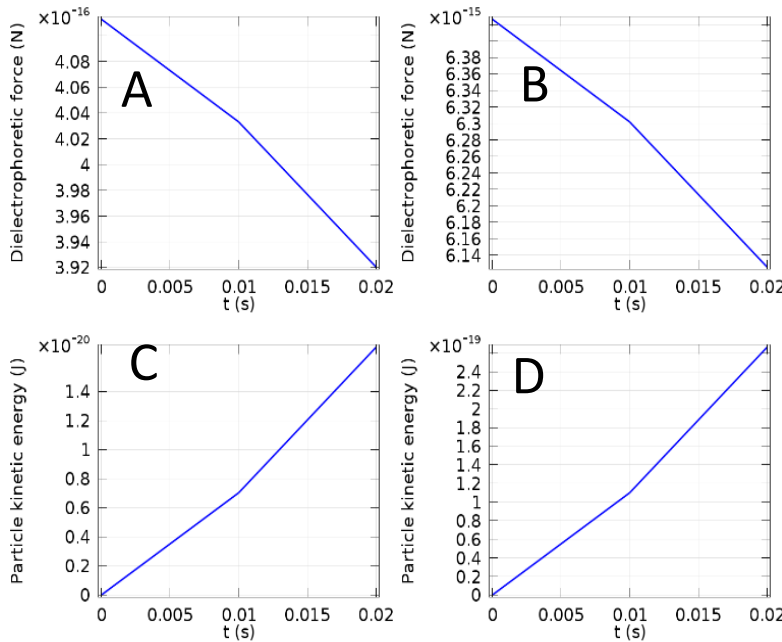

Figure 7: A comparison between the liver cancer cells and normal liver cells under effect of DEP rotation force in: A) the average induced DEP force on normal liver cells, B) the average induced DEP force on liver cancer cells, C) the average acquired kinetic energy by normal liver cells, D) the average acquired kinetic energy by liver cancer cells.
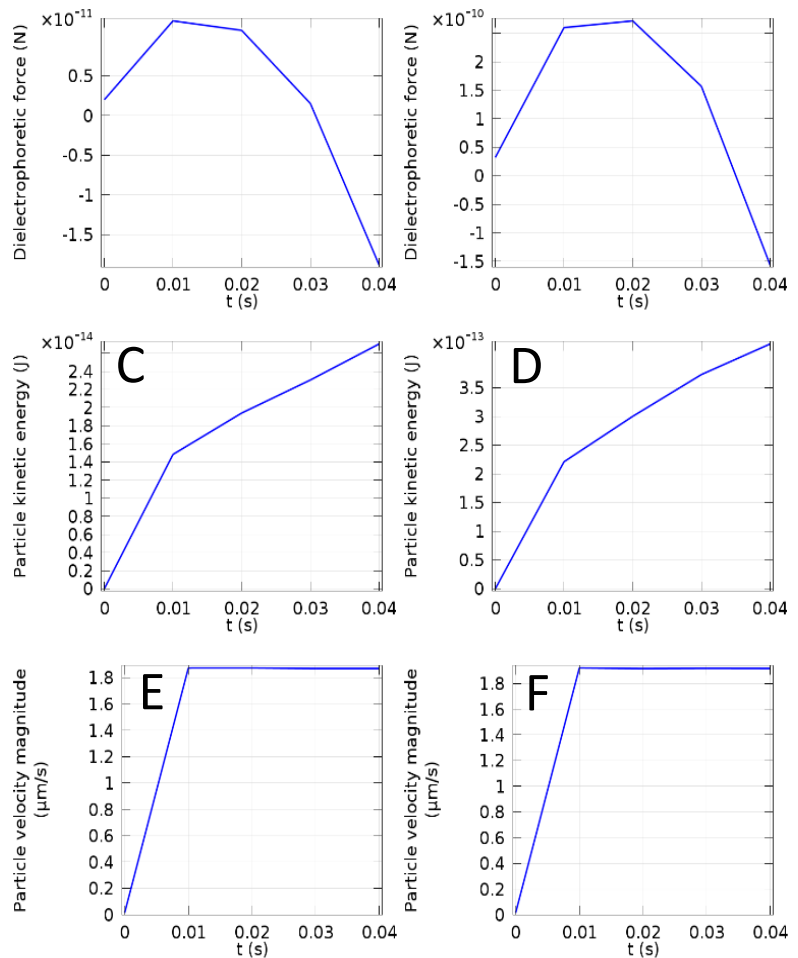

Figure 8: A comparison between the liver cancer cells and normal liver cells under effect of DEP levitation force in: A) the average induced DEP force on normal liver cells, B) the average induced DEP force on liver cancer cells, C) the average acquired kinetic energy by normal liver cells, D) the average acquired kinetic energy by liver cancer cells, E) the variance velocity of normal liver cells, and F) the variance velocity of liver cancer cells.
Each of Figure 6, Figure 7 and Figure 8 shows the follows:

- The induced DEP force on the liver cancer cells that is generated by the three configurations (traveling, electro-rotation and levitation) of the proposed electro-kinetic platform is greater than the induced DEP force on the normal liver cells.

- The acquired kinetic energy by the liver cancer cells that is generated by the three configurations (traveling, electro-rotation and levitation) of the proposed electro-kinetic platform is greater than The acquired kinetic energy by the normal liver cells.

- The variance velocity of the liver cancer cells that is generated by the two configurations (traveling and levitation) of the proposed electro-kinetic platform is slightly greater than the variance velocity of the normal liver cells.

Consequently, the proposed electro-kinetic platform able to characterize the liver cancer cells.

\section{CONCLUSION}

A 2D model of the three configurations of the proposed DEP electro-kinetic platform based on PCB technology for manipulation and characterization of liver cancer cells is presented and discussed. The simulation results show that the proposed electro-kinetic platform able to manipulate and differentiate the liver cancer cells from the normal liver cells by the comparison in the induced DEP force, the acquired kinetic energy and the velocity. The proposed electro-kinetic platform based on PCB technology is a good candidate to manipulate and characterize the different biological particles with high efficiency such as liver cancer cells. In the future work, the proposed electro-kinetic platform will be implemented to confirm the concept of the paper by experimental results.

\section{ACKNOWLEDGMENTS}

This research was partially funded by Zewail City of Science and Technology, AUC, the STDF, Intel, Mentor Graphics, ITIDA, SRC, ASRT and MCIT.

\section{REFERENCES}

[1] Pohl, H.A. and Pohl, H.A. Dielectrophoresis: the behavior of neutral matter in nonuniform electric fields. Cambridge: Cambridge university press, 1978, 80.

[2] Crane, J.S. and Pohl, H.A. A study of living and dead yeast cells using dielectrophoresis. Journal of the Electrochemical Society, 1968, 115(6), 584-586.

[3] Y. H. Ghallab and W. Badawy, Lab-on-a-Chip: Techniques, Circuits and Biomedical Applications Norwood, MA: Artech House, 2010.

[4] Barbaros C- etin, Dongqing Li, Review Dielectrophoresis in microfluidics Technology, Electrophoresis, 32, 2410-2427, 2011.

[5] M. Washizu, and O. Kurosawa "Electrostatic manipulation of DNA in microfabricated structures “, IEEE Transactions on Industry Applications, 1990, 26(6), 1165-1172.

[6] Y. H. Ghallab and W. Badawy "Sensing methods of Dielectrophorieses from Bulky instruments to Lab-on-achip", IEEE Circuit and Systems Magazine, 2004, Q3 issue, 4, 5-15. 
[7] A. Schmitz, S. Wagner, R. Hahn, H. Uzun and C. Hebling. Stability of planar PEMFC in printed circuit board technology. Journal of Power Sources, 2004, 127(1), 197-205.

[8] Ferlay J, Soerjomataram I, Ervik M, Dikshit R, Eser S, Mathers C, Rebelo M, Parkin DM, Forman D, Bray, F. GLOBOCAN 2012 v1.1, Cancer Incidence and Mortality Worldwide: IARC CancerBase No. 11 [Internet]. Lyon, France: International Agency for Research on Cancer; 2014. Available from:http://globocan.iarc.fr, accessed on 16/01/2015.

[9] L. Hartley, K.V.I.S. Kaler, J. Luo, R. Paul*, Discrete planar electrode dielectrophoresis systems, 0-7803-37165 IEEE, 1997.

[10] T. B. Jones. Basic theory of dielectrophoresis and electrorotation.Engineering in Medicine and Biology Magazine, IEEE, 2003, 22(6), 33-42.

[11] A. Boughriet, Z. Wu, H. McCann and L. E. Davis, The Measurement of Dielectric Properties of liquids at Microwave Frequencies Using Open-ended Coaxial Probes.

[12] Holczbauer A, Factor VM, Andersen JB, et al. Modeling Pathogenesis of Primary Liver Cancer in LineageSpecific Mouse Cell Types. Gastroenterology, Vol.145 (1): P.221-231, 2013.

[13] Joines WT, Zhang Y, Li C, and Jirtle RL., the measured electrical properties of normal and malignant human tissues from 50 to $900 \mathrm{MHz}$, Med phys. Vol. 21(4), P. 547-550, 1994

[14] Robert A. Freitas Jr., Nanomedicine, Volume I: Basic Capabilities, Landes Bioscience, Georgetown, TX, 1999.
[15] E. G. Cen, C. Dalton, Y. Li, S. Adamia, L.M. Pilarski and K. V. Kaler. A combined dielectrophoresis, traveling wave dielectrophoresis and electrorotation microchip for the manipulation and characterization of human malignant cells. Journal of microbiological methods, 2004, 58(3), 387-401.

[16] L. M. Fu, G.B. Lee, Y. H. Lin, and R. J. Yang, "Manipulation of microparticles using new modes of traveling wave dielectrophoretic force: Numerical simulation and experiments," Journal of IEEE/ASME Transactions (Mechatronics), Vol. 9 (2), P.377-383, 2004.

[17] S. I. Han, Y.D. Joo, and K. H. Han. An electrorotation technique for measuring the dielectric properties of cells with simultaneous use of negative quadrupolar dielectrophoresis and electrorotation. Analyst, 2013, 138 (5), 1529-1537.

[18] Elango, Iniyan Soundappa. Development of a Dielectrophoretic Chip for Single Cell Electrorotation. Diss. Arizona State University, 2012.

[19] Abdelbaset, R., Ghallab, Y.H., Abdelhamid, H., Ismail, Y. and El-Wakad, M.T., 2016, May. A 2D model of traveling wave Dielectrophoresis microelectrode array based on printed circuit board technology for manipulation and characterization of malignant and normal liver cells. InElectronics, Communications and Computers (JEC-ECC), 2016 Fourth International JapanEgypt Conference on (pp. 91-94). IEEE. 